\title{
Inventory control of service parts in the final phase
}

\author{
Ruud H. Teunter ${ }^{\text {a,* }}$, Willem K. Klein Haneveld ${ }^{\mathrm{b}}$ \\ ${ }^{a}$ Faculty of Economics, Econometric Institute, Erasmus University, P.O. Box 1738, NL-3000 DR Rotterdam, Netherlands \\ ${ }^{\mathrm{b}}$ Department of Econometrics, University of Groningen, P.O. Box 800, 9700 AV Groningen, Netherlands
}

Received 18 September 1999; accepted 14 March 2001

\begin{abstract}
We consider an appliance manufacturer's problem of controlling the inventory of a service part in its final phase. That phase begins when the production of the appliance containing that part is discontinued (time 0 ), and ends when the last service contract on that appliance expires. Thus, the planning horizon is deterministic and known. There is no setup cost for ordering. However, if a part is not ordered at time 0 , its price will be higher. The objective is to minimize the total expected undiscounted costs of replenishment, inventory holding, backorder, and disposal (of unused parts at the end of the planning horizon). We propose an ordering policy consisting of an initial order-up-to level at time 0 , and a subsequent series of decreasing order-up-to levels for various intervals of the planning horizon. We present a method of calculating the optimal policy, along with a numerical example and sensitivity analysis. (c) 2002 Elsevier Science B.V. All rights reserved.
\end{abstract}

Keywords: Inventory control; Service parts; Obsolescence

\section{Introduction}

We consider an appliance manufacturer's problem of controlling the inventory of a service part in its final phase. That phase begins when the production of the appliance containing that part is discontinued (time 0), and ends when the last service contract on that appliance expires. The final phase is, in general, the longest phase of the life cycle of a service part. In the electronics industry, for instance, the length of the final phase ranges from 4 years for small household appliances to 30 years for expensive medical equipment. The production of electronic appliances is normally stopped after less than 2 years (see [15]).

Two factors characterize inventory control of service parts in the final phase. First, there can be a considerable price increase after time 0 . Second, there is a large risk of obsolescence. The possible price

\footnotetext{
${ }^{*}$ Corresponding author. Tel.: +31-010-4081523; fax: +31-010-4089162.

E-mail addresses: teunter@yahoo.com (R.H. Teunter), w.k.klein.haneveld@eco.rug.nl (W.K. Klein Haneveld).
} 
increase results from the sudden decrease in demand for parts when the production of an appliance is discontinued (only the demand for service parts remains). We encountered situations in practice with price increases of more than 300\% (see [14]). The risk of obsolescence is evident, since ordered parts that remain unused at the end of the final phase have to be either recycled or disposed of.

In this paper, we propose an ordering policy for service parts that takes these two factors into account. There is a special order-up-to level at time 0 , in order to take advantage of the low price at that time. After time 0 , a series of decreasing order-up-to levels is applied for various intervals of the final phase, in order to reduce the risk of obsolescence.

The objective is to find the policy of this type that minimizes the total expected undiscounted cost of replenishment (no setup cost), inventory holding, backorder and disposal. We make the following assumptions. The planning horizon, i.e., the length of the final phase, is deterministic. That is, the time at which all stock becomes obsolete is known beforehand. At the end of the planning horizon, all remaining stock has to be either recycled or disposed of, and there is no recycling or disposing of parts before that time. There is a deterministic replenishment lead time. There is backordering. That is, demands that cannot be satisfied immediately are backordered until they can be satisfied. Demands that are still backordered at the end of the planning horizon are lost at that time. Demand follows a Poisson process with an intensity that remains constant throughout the entire planning horizon. Orders can be placed at any time, and there is continuous review.

The remainder of the paper is organized as follows. We start with literature reviews on the final phase and on obsolescence in Section 2. In Section 3 the model and the underlying assumptions are discussed, and the order-up-to policies are defined. In Sections 4 and 5 we determine the optimal order-up-to policy after time 0 . A numerical example together with substantial sensitivity analysis on the optimal policy after time 0 is discussed in Section 6. In Section 7 we derive an optimality condition for the optimal order-up-to level at time 0 . The limitations of our approach and directions for future extensions are discussed in Section 8 . We end with some conclusions in Section 9.

\section{Literature review}

\subsection{Final phase service parts logistics}

To the best of our knowledge, Fortuin [3,4], Geurts and Moonen [5], Klein Haneveld and Teunter [8], Teunter and Fortuin [15,16], and Teunter and Klein Haneveld [17], are the only authors who also focus on final phase service parts logistics. Except for Geurts and Moonen, all these authors discuss situations where it is impossible to order parts after the beginning of the final phase.

Geurts and Moonen [5] assume an increase in the setup cost for ordering (the price of an item remains unchanged) for parts that are ordered after the beginning of the final phase. They assume a zero replenishment lead time and a zero holding cost rate. Geurts and Moonen find optimal policies (numerically) for insurance type service parts by dividing the planning horizon into discrete periods of equal length and applying dynamic programming.

Klein Haneveld and Teunter [8] study almost the same model as we do, but assume a zero replenishment lead time and a fixed penalty each time a demand occurs and no part is in stock. As a result, the order-up-to level after the beginning of the final phase is either zero or one. In our model, as we will show, it can be $-\infty$ or have any non-negative discrete value. Furthermore, we will characterize the optimal policy in closedform formulas, whereas Klein Haneveld and Teunter [8] focus on a near-optimal policy.

Besides these authors, many others have studied the risk of obsolescence that characterizes the final phase of a service part. We summarize the most important results in the next subsection. 


\subsection{Obsolescence}

It is important to distinguish between perishability and obsolescence. We speak of perishability if parts that are held in stock worsen or can be ruined or destroyed. Perishability is also known as deterioration. Excellent overviews of perishability are given in $[9,11]$. We speak of obsolescence if there is a risk of parts becoming obsolete, i.e., going out of use, due to a change in the environment. With respect to inventory control of service parts in the final phase there is a risk of obsolescence, since all parts remaining at the end of the final phase become useless. Another example of obsolescence is that resulting from the drop in demand when a competitor enters the market with a low-priced substitute. In the first example, the moment of obsolescence is known in advance, at least approximately, whereas it comes as a surprise in the second. We refer to these situations as expected obsolescence and sudden obsolescence, respectively. In the remainder of this section, we will review the results on expected obsolescence. For a review of sudden obsolescence, we refer to [18, Section 2.2.2].

Consider the 'classical' discrete time continuous review inventory model with constant setup cost for ordering, linear holding and backorder cost, and a deterministic replenishment lead time. Continuous review in a discrete time model means that the inventory position is reviewed at either the beginning or the end of each period. Scarf [12] shows, using a dynamic programming formulation of the model, that for a finite planning horizon, an $(s, S)$ net stock order-up-to policy is optimal for each period. Scarf [12] also shows that an $(S-1, S)$ order-up-to policy is optimal if the setup cost for ordering is zero. Generalizations of these results are given by several authors, including Iglehart [6,7], Porteus [10], Tijms [19], and Veinnot [20]. In [2] a general form of the theorem on optimality of order-up-to policies is given and proven. This theorem includes the discrete time version of the model that is studied in this paper, that is, with backordering, with linear holding, ordering, and backordering cost, and with a deterministic planning horizon and lead time.

Barbosa and Friedman [1] analyze a continuous time continuous review inventory problem with a deterministic demand rate that is continuously decreasing and reaches zero at the end of the finite planning horizon. They assume that the variable replenishment cost is 0 , i.e., only a fixed setup cost is incurred when an order is placed. So the risk of obsolescence, interpreted as the risk of spending money on parts that are never needed, is ignored.

As aforementioned, the model studied in this paper is closely related to the classical model discussed above. In fact, except for the price increase after time 0 , it is a special case of that model with no setup cost for ordering. However, we model time continuous, whereas time is modeled discrete in the classical model. As we will show, the continuous time modeling allows us to characterize optimal parameters of policies by closed-form expressions. For the classical discrete time model, dynamic programming techniques are needed in order to calculate optimal policies.

\section{Model}

The goal is to find an ordering policy that minimizes the total expected (undiscounted) cost during the finite planning horizon. We make the following modeling assumptions:

1. Time is modeled as a continuous variable $t \in[0, P] . P$ is the finite planning horizon.

2. The demand for the part that is under consideration is stationary, and follows a Poisson process with intensity $\lambda>0$ in the time period $[0, P]$.

3. Service parts are held in stock. In order to increase the stock, additional service parts may be ordered at any time $t \in[0, P]$. Ordered parts arrive after a non-negative deterministic lead time $L$.

4. No parts are disposed of before the end of the planning horizon $P$. All stock remaining at the end of the planning horizon is disposed of at that time. 
5. When a demand occurs, it is satisfied immediately if possible. If the stock on hand is zero, any demand before time $P$ is fully backordered. At time $P$ backorders, if there are any, are considered as lost demands.

6. The following costs are incurred:

Replenishment cost: If $k$ service parts are ordered at time 0 , the replenishment cost is $k$. That is, we normalize the cost parameters, such that the price of a part at time 0 is equal to 1 . If $k$ service parts are ordered at any time $t>0$, the replenishment cost is $c k$ with $c \geqslant 1$. So, there is no ordering (or setup) cost, and service parts may be less expensive at time 0 .

Holding cost: A holding cost rate $h>0$ is incurred during the time that a part is held in stock.

Backorder cost: A backorder cost rate $p>0$ is incurred during the time that a demand is backordered. Disposal cost: If $k$ service parts are disposed of at time $P$, the disposal cost is $d k$, where the unit disposal cost $d>-c$. The value of $d$ can be negative if the remaining parts are (partly) recycled.

We restrict our attention to (net stock) order-up-to policies where the order-up-to level $S(t)$ may depend on the time $t$. Recall from Section 2.2 that this class of time-dependent order-up-to policies contain all optimal policies for the classical discrete time inventory model. As argued earlier, our model is the continuous time analogy of the classical discrete time model. It therefore seems reasonable to assume that the class of time-dependent order-up-to policies also contains all optimal policies for our model.

We restrict ourselves to integer-valued order-up-to levels $S(t)$. This is without loss of generality, since the demands are integer-valued (always 1). Moreover, since it is not reasonable to order any parts after time $P-L$, we set $S(t)=-\infty$ for $t \in[P-L, P]$. For $t<P-L$ we take $S(t)$ either $-\infty$ or non-negative, since finite negative values cannot be optimal. Indeed, suppose that the net stock is zero, when at time $t<P-L$ a demand occurs. There are two sensible options. Either an order is placed immediately and the demand will be satisfied at time $t+L<P$ or the demand will be backordered until time $P$, and will never be satisfied. There is no sense in postponing the order until some time after $t$, since that will result in a higher shortage cost, whereas all other costs remain the same. If the first option is better than the second option, i.e., if the backorder cost in period $[t+L, P]$ is smaller than $c$, then the optimal order-up-to level is at least 0 . Otherwise, it is better not to order and let the stock drop to -1 . However, using the same argument, it will then also be optimal to let the stock drop to -2 if a demand occurs at time $t$ and the net stock just before that time is -1 . Repeating this argument gives that the optimal order-up-to level at time $t$ is either non-negative or $-\infty$. It is also reasonable to expect that $S(t)$ is non-increasing in $t$, since the demand is stationary and the cost parameters do not depend on time.

The optimal value of $S(t)$ will depend on the parameters of the problem, of course. Because the demand process and the specification of the costs do not depend on time, the dependence of $S(t)$ on the planning horizon $P$ is simple: for all $P>0$ and all $t \in(0, P]$ it only depends on the remaining time $P-t$. Indeed, the problem specification is such that the subproblem from time $t$ onward is equivalent to the problem after time 0 , if the planning horizon is reduced to $P-t$. So, we restrict the attention to order-up-to levels given by

$$
S(t)=H(P-t), \quad t \in(0, P],
$$

where $H$ is a non-decreasing integer-valued function on $[0, \infty)$, not depending on $P$, which is either $-\infty$ or non-negative. It is to be expected that for any fixed $t$

$$
S(t) \leqslant \lim _{P \rightarrow \infty} S(t)=\lim _{u \rightarrow \infty} H(u)=: \bar{S}
$$

is finite, where $\bar{S}$ is the order-up-to level of the infinite horizon version of the model. Consequently, the function $H$ has only finite many jumps, so that it can be described by finite many parameters $U_{k}$ :

$$
U_{k}:=\inf _{u}\{u: H(u) \geqslant k\}, \quad k=0,1, \ldots, \bar{S},
$$

satisfying $L \leqslant U_{0} \leqslant U_{1} \leqslant \cdots \leqslant U_{\bar{S}}<\infty$. This is illustrated in Fig. 1. It will transpire to be useful to describe $H$ in terms of the non-negative parameters $L_{0}, L_{1}, \ldots, L_{\bar{S}}$, where 


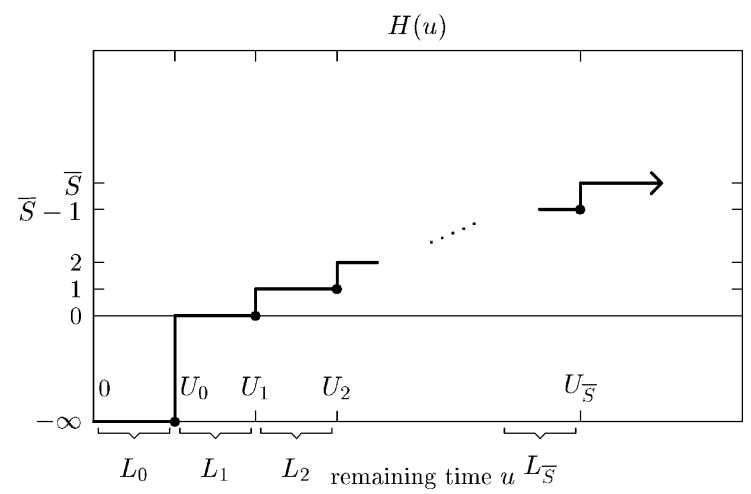

Fig. 1. Optimal order-up-to level after time 0 for any value of the remaining time until the end of the planning horizon.

$$
L_{0}:=U_{0} \quad \text { and } \quad L_{i}:=U_{i}-U_{i-1}, \quad i=1,2, \ldots, \bar{S}
$$

so that

$$
U_{i}:=\sum_{j=0}^{i} L_{j}, \quad i=0,1, \ldots, \bar{S} .
$$

The parameters $L_{0}, L_{1}, L_{2}, \ldots, L_{\bar{S}}$ are called order-up-to times, since they represent the length of the time period during which the optimal order-up-to level is $-\infty, 0,1, \ldots, \bar{S}-1$, respectively. This is also illustrated in Fig. 1.

Let us summarize the above discussion on time-dependent order-up-to policies. We restrict our attention to so-called remaining time order-up-to policies. Such a policy is denoted by $\pi\left(S_{0}, L_{0}, L_{1}, \ldots, L_{\bar{S}}\right)$, since it is specified completely by these parameters in the following way:

- At time 0 , the order-up-to level is $S_{0}$.

- At time $t \in\left(0, P-L_{0}\right)$, the order-up-to level is $k \in\{0,1, \ldots, \bar{S}-1\}$, if the remaining time $P-t$ satisfies $\sum_{j=0}^{k} L_{j}<P-t \leqslant \sum_{j=0}^{k+1} L_{j}$, that is if $P-\sum_{j=0}^{k+1} L_{j} \leqslant t<P-\sum_{j=0}^{k} L_{j}$.

- At time $t \in\left(0, P-L_{0}\right)$, the order-up-to level is $\bar{S}$, if the remaining time $P-t$ satisfies $P-t>\sum_{j=0}^{\bar{S}} L_{j}$, that is if $t<P-\sum_{j=0}^{S} L_{j}$.

- At time $t \in\left[P-L_{0}, P\right]$, no parts are ordered.

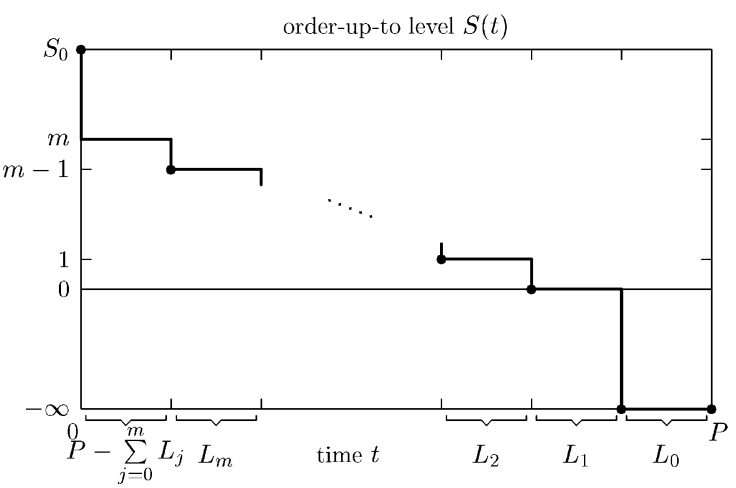

Fig. 2. Remaining time order-up-to policy $\pi\left(S_{0}, L_{0}, L_{1}, \ldots, L_{\bar{S}}\right)$, with initial order-up-to level $S_{0}$ and order-up-to times $L_{0}, L_{1}, \ldots, L_{\bar{S}}$, when the planning horizon is $P$. 
See Fig. 2. Notice that the maximum value of the order-up-to level is $m^{\prime}$, determined by $\sum_{j=0}^{m^{\prime}} L_{j}<P \leqslant \sum_{j=0}^{m^{\prime}+1} L_{j}$, and that the parameters $L_{m^{\prime}+1}, \ldots, L_{\bar{S}}$ are irrelevant.

In what follows, we determine optimal remaining time order-up-to policies by calculating optimal values for the parameters involved. First of all, $\bar{S}$ is calculated in Section 4. Then, recursively, the values of $L_{0}, L_{1}, \ldots, L_{\bar{S}}$ are optimized in Section 5 . This is done by successively increasing $P$, using the fact that $L_{0}, L_{1}, \ldots, L_{\bar{S}}$ do not depend on $P$. Finally, $S_{0}$ is optimized in Section 7.

\section{Characterization of $\bar{S}$}

In this section we determine $\bar{S}$. Recall that $\bar{S}$ is the optimal order-up-to level after the initial provisioning if the remaining time until the end of the planning horizon is infinite. That is, it is the optimal order-up-to level in the infinite horizon model equivalent to the one presented in Section 3, i.e., with $P$ set to $\infty$. We remark that a characterization of $\bar{S}$, as we will present next, has been discussed by many other authors, see for instance [13].

Clearly, in the infinite horizon situation, an order-up-to policy with a constant order-up-to level $\bar{S}$ is optimal, since all parameters of the model are stationary. Consider any time $t>L$ in such an infinite horizon situation. The stock on hand at time $t$ is equal to the net stock at time $t-L$ minus the demand in period $(t-L, t]$. Hence, the average cost rate (at any time $t>L$ ) associated with order-up-to level $S, S=0,1, \ldots$, is

$$
f(S):=h E(S-D)^{+}+p E(S-D)^{-},
$$

where $D$ is the random variable corresponding to the demand during a time period of length $L$. That is, $D$ is Poisson distributed with parameter $\lambda L$. As is well known from the classical newsboy problem, $f$ is a convex function, that is minimal for $S$ equal to the $p /(p+h)$ quantile of the distribution function $Q$ of $D$. Hence, we can characterize the optimal order-up-to level $\bar{S}$ as follows:

$$
\bar{S}:=\min \left\{k \in\{0,1, \ldots\}: Q(k, \lambda L) \geqslant \frac{p}{p+h}\right\}
$$

where

$$
Q(k, \mu)=\sum_{i=0}^{k} \frac{\mathrm{e}^{-\mu} \mu^{i}}{i !}
$$

for all $k=0,1, \ldots$ and $\mu>0$. We note that if $Q(\bar{S}, \lambda L)>p /(p+h)$, then $\bar{S}$ is the unique average cost minimizer; if $Q(\bar{S}, \lambda L)=p /(p+h)$, both $\bar{S}$ and $\bar{S}+1$ are the minimizers.

\section{Optimal order-up-to times}

In this section, successively for $i=0,1, \ldots, \bar{S}$, we derive an optimality condition for $L_{i}$ given $L_{0}^{*}, \ldots, L_{i-1}^{*}$. Our approach is based on the following observation. Let $\pi\left(S_{0}^{*}, L_{0}^{*}, \ldots, L_{\bar{S}}^{*}\right)$ be any optimal policy. Using this policy, the order-up-to level $S(t)$ drops from $i$ to $i-1$ (to $-\infty$ if $i=0$ ) at time $t_{i}$, where

$$
t_{i}:=P-\sum_{j=0}^{i} L_{j}^{*},
$$


at least if $P>\sum_{j=0}^{i} L_{j}^{*}$ what we assume. We will study a marginal perturbation of $t_{i}$. That is, for a small $\epsilon>0$ we compare the policies

$$
\begin{aligned}
& \pi_{i}^{*}(\epsilon):=\pi\left(S_{0}^{*}, L_{0}^{*}, \ldots, L_{i-1}^{*}, L_{i}^{*}-\epsilon, L_{i+1}^{*}+\epsilon, L_{i+2}^{*}, \ldots, L_{\bar{S}}^{*}\right), \\
& \pi_{i}^{*}(-\epsilon):=\pi\left(S_{0}^{*}, L_{0}^{*}, \ldots, L_{i-1}^{*}, L_{i}^{*}+\epsilon, L_{i+1}^{*}-\epsilon, L_{i+2}^{*}, \ldots, L_{\bar{S}}^{*}\right) .
\end{aligned}
$$

These policies have the same order-up-to levels $S(t)$, except in the time interval $\left[t_{i}-\epsilon, t_{i}+\epsilon\right)$; then the first one uses level $i$ and the second one uses level $i-1$. See Fig. 3. What is the effect of these marginal perturbations? Obviously, both policies $\pi_{i}^{*}(\epsilon)$ and $\pi_{i}^{*}(-\epsilon)$ imply the same decisions and therefore the same cost unless the realization of the demand process is such that the event $E_{i}(\epsilon)$ occurs

$$
E_{i}(\epsilon):=\text { in time interval }\left[t_{i}-\epsilon, t_{i}+\epsilon\right) \text { the net stock becomes } i \text { and a part is demanded. }
$$

When $E_{i}(\epsilon)$ happens, $\pi_{i}^{*}(\epsilon)$ orders a part in $\left[t_{i}-\epsilon, t_{i}+\epsilon\right)$ whereas $\pi_{i}^{*}(-\epsilon)$ does not. In that case also future decisions of $\pi_{i}^{*}(\epsilon)$ may differ from those of $\pi_{i}^{*}(-\epsilon)$, but before time $t_{i}-\epsilon$ both policies agree, of course. Therefore, the total expected cost under $\pi_{i}^{*}(\epsilon)$ minus the total expected cost under $\pi_{i}^{*}(-\epsilon)$ is equal to $\operatorname{Pr}\left(E_{i}(\epsilon)\right) \Delta_{i}^{*}(\epsilon)$, where

$$
\Delta_{i}^{*}(\epsilon):=E_{\pi_{i}^{*}(\epsilon)}\left[\text { total cost in }\left[t_{i}-\epsilon, P\right] \mid E_{i}(\epsilon)\right]-E_{\pi_{i}^{*}(-\epsilon)}\left[\text { total cost in }\left[t_{i}-\epsilon, P\right] \mid E_{i}(\epsilon)\right] .
$$

Now let $\epsilon \downarrow 0$. Then $\operatorname{Pr}\left(E_{i}(\epsilon)\right)$ converges to 0 . More importantly, we claim that $\Delta_{i}^{*}(\epsilon)$ converges to 0 too, that is,

$$
\Delta_{i}^{*}(0+):=\lim _{\epsilon \downarrow 0} \Delta_{i}^{*}(\epsilon)=0 .
$$

The idea is, that for the restriction of the problem to the remaining period of time $\left[t_{i}, P\right]$ the policy $\pi^{*}$ must also be optimal, for all values of the initial stock at time $t_{i}$. This observation leads us to the following approach for finding the optimal value $L_{i}^{*}$ for $L_{i}$ given $L_{0}^{*}, \ldots, L_{i-1}^{*}$. For any fixed $L_{i}$, we consider the problem on the time interval $\left[t_{i}, P\right]$, where

$$
t_{i}:=P-\sum_{j=0}^{i-1} L_{j}^{*}-L_{i}
$$

is supposed to be positive. It is assumed that the net stock just before time $t_{i}$ is equal to $i$, and that a demand occurs at time $t_{i}$. Two policies are being compared, $\pi_{i}^{+}$and $\pi_{i}^{-}$. Both use the order-up-to levels $S(t)$ determined by $L_{0}^{*}, \ldots, L_{i-1}^{*}$, and $L_{i}$ at all times $t>t_{i}$. At time $t_{i}$ they are different

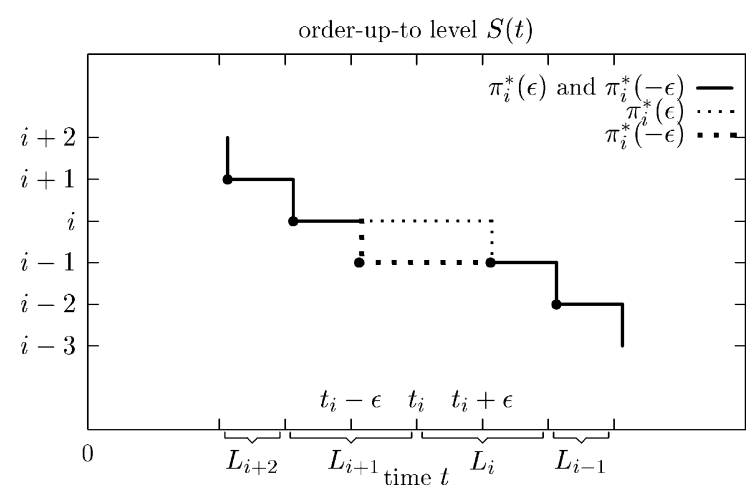

Fig. 3. Optimal order-up-to level after time 0 under policies $\pi_{i}^{*}(\epsilon)$ and $\pi_{i}^{*}(-\epsilon)$. 
- $\pi_{i}^{+}$uses level $i$ at time $t_{i}$, hence an order is placed at time $t_{i}$.

- $\pi_{i}^{-}$uses level $i-1(-\infty$ if $i=0)$ at time $t_{i}$, hence no order is placed at time $t_{i}$.

Define

$$
\Delta_{i}\left(L_{i}\right):=\text { the expected total cost of } \pi_{i}^{+} \text {in }\left[t_{i}, P\right]-\text { the expected total cost of } \pi_{i}^{-} \text {in }\left[t_{i}, P\right] .
$$

Then the observations above indicate that for $L_{i}=L_{i}^{*}$ we get $\Delta_{i}\left(L_{i}^{*}\right)=\Delta_{i}^{*}(0+)=0$. So a necessary optimality condition for $L_{i}$ is

$$
\Delta_{i}\left(L_{i}\right)=0 \text { if } L_{i}=L_{i}^{*} .
$$

That is, if $L_{i}=L_{i}^{*}$ then at the point of time where the order-up-to level drops from $i$ to $i-1$ it does not matter if one uses $i$ or $i-1$, even if it is given that the net stock level is equal to $i$ and a demand occurs. On the other hand, under these circumstances it might be expected that $\pi_{i}^{-}$is better than $\pi_{i}^{+}$if $L_{i}<L_{i}^{*}$ and the other way around if $L_{i}>L_{i}^{*}$, that is,

$$
\begin{array}{ll}
\Delta_{i}\left(L_{i}\right)>0 & \text { if } L_{i}<L_{i}^{*}, \\
\Delta_{i}\left(L_{i}\right)<0 & \text { if } L_{i}>L_{i}^{*} .
\end{array}
$$

If this transpires to be true, the necessary optimality condition is sufficient too.

In Sections 5.1 and 5.2 we will derive explicit formulas for $\Delta_{i}\left(L_{i}\right)$, successively for $i=0,1, \ldots, \bar{S}$. It transpires that for each $i=0,1, \ldots, \bar{S}$, the function $\Delta_{i}\left(L_{i}\right)$ is continuously decreasing from a positive value for $L_{i}=0$ to a negative value for $L_{i} \rightarrow \infty$, so that the equation $\Delta_{i}\left(L_{i}\right)=0$ has a unique solution that must be $L_{i}^{*}$.

\subsection{Calculation of $L_{0}^{*}$}

For any $L_{0}>0$, consider the inventory system in the period $\left[t_{0}, P\right]$, where $t_{0}=P-L_{0}>0$. Assume that the net stock just before time $t_{0}$ is zero, and that a demand occurs at time $t_{0}$. We compare the policies:

$$
\begin{aligned}
& \pi_{0}^{+}: S(t)=0 \text { if } t=t_{0}, S(t)=-\infty \text { for all } t>t_{0}, \\
& \pi_{0}^{-}: S(t)=-\infty \text { for all } t \geqslant t_{0} .
\end{aligned}
$$

That is, $\pi_{0}^{+}$orders a new part at time $t_{0}$, and orders no parts later, whereas $\pi_{0}^{-}$does not order any part at or after time $t_{0}$. By ordering a part at $t_{0}$, a shortage is avoided during a time interval of length $P-\min \left(P, P-L_{0}+L\right)=\left(L_{0}-L\right)^{+}$, so that the difference in expected cost between policies $\pi_{0}^{+}$and $\pi_{0}^{-}$is

$$
\Delta_{0}\left(L_{0}\right)=c-p\left(L_{0}-L\right)^{+} .
$$

Hence, $L+(c / p)$ is the unique value for $L_{0}$, for which policies $\pi_{0}^{+}$and $\pi_{0}^{-}$give the same expected cost, so that

$$
L_{0}^{*}=L+\frac{c}{p} .
$$

\subsection{Calculation of $L_{i}^{*}, i=1,2, \ldots, \bar{S}$}

In order to characterize the optimal value $L_{i}^{*}, i=1,2, \ldots, \bar{S}$, we proceed as follows. We assume that $L_{0}^{*}, \ldots, L_{i-1}^{*}$ have been determined already.

For any $L_{i}>0$, consider the inventory system in period $\left[t_{i}, P\right]$, where $t_{i}=P-\sum_{j=1}^{i-1} L_{j}^{*}-L_{i}>0$. Assume that the net stock just before time $t_{i}$ is $i$, and that a demand occurs at time $t_{i}$. We compare the policies: 
- $\pi_{i}^{+}$: Order a new part at time $t_{i}$, restoring the net stock to $i$ at that time, and apply the policy $\pi\left(S_{0}, L_{0}^{*}, L_{1}^{*}, \ldots, L_{i-1}^{*}\right)$ for all $t>t_{i}$,

- $\pi_{i}^{-}$: Apply the policy $\pi\left(S_{0}, L_{0}^{*}, L_{1}^{*}, \ldots, L_{i-1}^{*}\right)$ for all $t \geqslant t_{i}$.

Note that the value of $S_{0}$ is not relevant for this comparison, since $t_{i}>0$.

The difference in expected cost between policies $\pi_{i}^{+}$and $\pi_{i}^{-}$is

$$
\begin{aligned}
\Delta_{i}\left(L_{i}\right) & =c \operatorname{Pr}\left(\mathscr{E}_{1}\right)+d \operatorname{Pr}\left(\mathscr{E}_{2}\right)+h E\left[\mathscr{T}_{1}\right]-p E\left[\mathscr{T}_{2}\right] \\
& =c \operatorname{Pr}\left(\mathscr{E}_{1}\right)+d \operatorname{Pr}\left(\mathscr{E}_{2}\right)+(h+p) E\left[\mathscr{T}_{1}\right]-p E\left[\mathscr{T}_{1}+\mathscr{T}_{2}\right],
\end{aligned}
$$

where the events $\mathscr{E}_{1}, \mathscr{E}_{2}$ and the random variables $\mathscr{T}_{1}, \mathscr{T}_{2}$ are defined as follows:

- The increase in the total number of orders in $\left[t_{i}, P\right]$ is either 0 or 1 . The event that it is equal to one is denoted by $\mathscr{E}_{1}$.

- The increase in the total number of disposed parts at the end of the planning horizon is either 0 or 1 . The event that it is equal to one is denoted by $\mathscr{E}_{2}$.

- At any time $t \in\left[t_{i}, P\right]$ the stock on hand increases by 1 or does not change. We define $\mathscr{T}_{1}$ as the random variable denoting the length of the time interval during which the stock on hand is increased by 1 .

- At any time $t \in\left[t_{i}, P\right]$ the number of backorders decreases by 1 or does not change. We define $\mathscr{T}_{2}$ as the random variable denoting the length of the time interval during which the number of backorders is decreased by 1 .

In [18, Section 5.2], we present detailed derivations of the probabilities associated with events $\mathscr{E}_{1}$ and $\mathscr{E}_{2}$, and of the expected values of $\mathscr{T}_{1}$ and $\mathscr{T}_{2}$, under the conditions that the net stock just before time $t_{i}$ is $i$, and that a demand occurs at time $t_{i}$. We will use the results of those derivations, that are omitted here, to derive closed-form formulas for $L_{1}^{*}$ in Section 5.2.1, and for $L_{i}^{*}, i=2,3, \ldots, \bar{S}$ in Section 5.2.2.

\subsubsection{Calculation of $L_{1}^{*}$}

Knowing $L_{0}^{*}$ (see (3)) we now calculate $L_{1}^{*}$ under the assumption that the parameters of the problem are such that $\bar{S} \geqslant 1$, that is, $p-(h+p) \mathrm{e}^{-\lambda L}>0$ (see (2)).

For $i=1$ we have (see [18])

$$
\begin{aligned}
& \operatorname{Pr}\left(\mathscr{E}_{1}\right)=\mathrm{e}^{-\lambda L_{1}}, \\
& \operatorname{Pr}\left(\mathscr{E}_{2}\right)=\mathrm{e}^{-\lambda L_{1}} \mathrm{e}^{-\lambda L_{0}^{*},} \\
& \lambda E\left[\mathscr{T}_{1}\right]=\mathrm{e}^{-\lambda L}-\mathrm{e}^{-\lambda L_{1}} \mathrm{e}^{-\lambda L_{0}^{*}}, \\
& \lambda E\left[\mathscr{T}_{1}+\mathscr{T}_{2}\right]=1-\mathrm{e}^{-\lambda L_{1}}+\mathrm{e}^{-\lambda L_{1}} \lambda\left(L_{0}^{*}-L\right) .
\end{aligned}
$$

Hence, (4) gives for $i=1$,

$$
\lambda \Delta_{1}\left(L_{1}\right)=\mathrm{e}^{-\lambda L_{1}}\left(\lambda c+\lambda d \mathrm{e}^{-\lambda L_{0}^{*}}-(h+p) \mathrm{e}^{-\lambda L_{0}^{*}}+p-\lambda p\left(L_{0}^{*}-L\right)\right)+\left((h+p) \mathrm{e}^{-\lambda L}-p\right) .
$$

Using (3) this can be rewritten as

$$
\lambda \Delta_{1}\left(L_{1}\right)=\mathrm{e}^{-\lambda L_{1}}\left(\gamma_{1}+\beta_{1}\right)-\beta_{1},
$$

where

$$
\begin{aligned}
& \beta_{1}=p-(h+p) \mathrm{e}^{-\lambda L}, \\
& \gamma_{1}=\lambda d \mathrm{e}^{-\lambda L_{0}^{*}}+(h+p) \mathrm{e}^{-\lambda L}\left(1-\mathrm{e}^{-\lambda\left(L_{0}^{*}-L\right)}\right) .
\end{aligned}
$$

Because of $\bar{S} \geqslant 1$ we know that $\beta_{1}>0$, and because of (3) also $\gamma_{1}>0$. Therefore, $\lambda \Delta_{1}\left(L_{1}\right)$ is continuously decreasing on $[0, \infty)$ from the positive value $\gamma_{1}$ for $L_{1}=0$ to the negative value $-\beta_{1}$ for $L_{1} \rightarrow \infty$, so that the optimal value $L_{1}^{*}$ for $L_{1}$ is given by $\lambda \Delta_{1}\left(L_{1}^{*}\right)=0$, that is, 


$$
\lambda L_{1}^{*}=\ln \left(1+\frac{\gamma_{1}}{\beta_{1}}\right)
$$

Using (3) this gives

$$
\lambda L_{1}^{*}=\ln \left(1+\frac{\lambda d \mathrm{e}^{-\lambda(L+(c / p))}+(h+p) \mathrm{e}^{-\lambda L}\left(1-\mathrm{e}^{-\lambda(c / p)}\right)}{p-(h+p) \mathrm{e}^{-\lambda L}}\right) .
$$

As expected, on the boundary between $\bar{S}=0$ and $\bar{S}=1$, i.e., if $\mathrm{e}^{\lambda L} p /(p+h)=1$, we have $L_{1}^{*}=\infty$.

\subsubsection{Calculation of $L_{i}^{*}, i=2,3, \ldots, \bar{S}$}

Fix $i \geqslant 2$ and assume that $\lambda, p$ and $h$ are such that $\bar{S} \geqslant i$. Then we get (see [18])

$$
\begin{aligned}
& \operatorname{Pr}\left(\mathscr{E}_{1}\right)=B_{i} \mathrm{e}^{-\lambda L_{i}}, \\
& \operatorname{Pr}\left(\mathscr{E}_{2}\right)=C_{i} \mathrm{e}^{-\lambda L_{i}}, \\
& \lambda E\left[\mathscr{T}_{1}\right]=D_{i}+E_{i} \mathrm{e}^{-\lambda L_{i}}, \\
& \lambda E\left[\mathscr{T}_{1}+\mathscr{T}_{2}\right]=1+\left(A_{i}-1\right) \mathrm{e}^{-\lambda L_{i}},
\end{aligned}
$$

where

$$
\begin{aligned}
& A_{i}=\sum_{k=2}^{i} \sum_{n_{1, k} \in V_{k}[0]}\left[\left(\prod_{j=2}^{k} q\left(n_{j}, \lambda L_{i+1-j}^{*}\right)\right) k\right]+\sum_{n_{i} \in V_{i+1}[0]}\left[\left(\prod_{j=2}^{i} q\left(n_{j}, \lambda L_{i+1-j}^{*}\right)\right)\left(\lambda\left(L_{0}^{*}-L\right)+\sum_{j=2}^{i} n_{j}\right)\right], \\
& B_{i}=\sum_{n_{1, i} \in V_{i+1}[0]}\left[\prod_{j=2}^{i} q\left(n_{j}, \lambda L_{i+1-j}^{*}\right)\right] \text {, } \\
& C_{i}=\sum_{n_{1, i} \in V_{i+1}[0]}\left[\left(\prod_{j=2}^{i} q\left(n_{j}, \lambda L_{i+1-j}^{*}\right)\right) Q\left(i-1-\sum_{j=2}^{i} n_{j}, \lambda L_{0}^{*}\right)\right] \\
& D_{i}=i Q(i, \lambda L)-\lambda L Q(i-1, \lambda L)-(i-1) Q(i-1, \lambda L)+\lambda L Q(i-2, \lambda L) \text {, } \\
& E_{i}=(i-1) Q(i-1, \lambda L)-\lambda L Q(i-2, \lambda L) \\
& -\sum_{k=2}^{i-1} \sum_{n_{1, k} \in V_{k}[0]}\left(\prod_{j=2}^{k} q\left(n_{j}, \lambda L_{i+1-j}^{*}\right)\right)\left(\begin{array}{c}
(i-k) Q(i-k, \lambda L) \\
-\lambda L Q(i-k-1, \lambda L)
\end{array}\right) \\
& -\sum_{n_{1, i} \in V_{i+1}[0]}\left(\prod_{j=2}^{i} q\left(n_{j}, \lambda L_{i+1-j}^{*}\right)\right)\left(\begin{array}{c}
\left(i-\sum_{j=2}^{i} n_{j}\right) Q\left(i-\sum_{j=2}^{i} n_{j}, \lambda L_{0}^{*}\right) \\
-\lambda L_{0}^{*} Q\left(i-1-\sum_{j=2}^{i} n_{j}, \lambda L_{0}^{*}\right)
\end{array}\right), \\
& n_{1, k}=\left(n_{1}, \ldots, n_{k}\right), \quad k=1,2, \ldots, i, \\
& V_{k}[b]=\left\{\sum_{l=1}^{j} n_{l}<j+b \text { for } j=1, \ldots, k-1, \text { and } \sum_{l=1}^{k} n_{l} \geqslant k+b\right\}, \quad k=1, \ldots, i ; \quad b=0,1, \ldots, \\
& V_{i+1}[b]=\left\{\sum_{l=1}^{j} n_{l}<j+b \text { for } j=1, \ldots, i\right\}, \quad b=0,1, \ldots, \\
& q(k, \mu)=\frac{\mathrm{e}^{-\mu} \mu^{k}}{k !}, \quad k=0,1, \ldots ; \mu>0, \\
& Q(k, \mu)=\sum_{l=0}^{k} \frac{\mathrm{e}^{-\mu} \mu^{l}}{l !}, \quad k=0,1, \ldots ; \mu>0 .
\end{aligned}
$$


The numbers $A_{i}, \ldots, E_{i}$ depend on the parameters of the model, and on $L_{0}^{*}, L_{1}^{*}, \ldots, L_{i-1}^{*}$, but not on $L_{i}$. Hence we get

$$
\lambda \Delta_{i}\left(L_{i}\right)=\mathrm{e}^{-\lambda L_{i}}\left(\gamma_{i}+\beta_{i}\right)-\beta_{i},
$$

where

$$
\begin{aligned}
& \beta_{i}=p-(h+p) D_{i}, \\
& \gamma_{i}=\lambda c B_{i}+\lambda d C_{i}+(h+p)\left(D_{i}+E_{i}\right)-p A_{i} .
\end{aligned}
$$

If both $\beta_{i}>0$ and $\gamma_{i}>0$ are positive, then $\lambda \Delta_{i}\left(L_{i}\right)$ is continuously decreasing on $[0, \infty)$ from the positive value $\gamma_{i}$ for $L_{i}=0$ to the negative value $-\beta_{i}$ for $L_{i} \rightarrow \infty$, so that the optimal value $L_{i}^{*}$ for $L_{i}$ is given by $\lambda \Delta_{i}\left(L_{i}^{*}\right)=0$, that is,

$$
\lambda L_{i}^{*}=\ln \left(1+\frac{\gamma_{i}}{\beta_{i}}\right)
$$

or

$$
\lambda L_{i}^{*}=\ln \left(1+\frac{\lambda c B_{i}+\lambda d C_{i}+(h+p)\left(D_{i}+E_{i}\right)-p A_{i}}{p-(h+p) D_{i}}\right) .
$$

It remains to verify that both $\beta_{i}$ and $\gamma_{i}$ are positive, indeed. Unfortunately, we are not able do this analytically. We did, however, calculate $\beta_{i}$ and $\gamma_{i}$ in a large number of examples, and in each example both numbers turned out to be positive.

\subsection{Characterization of the optimal order-up-to policy after the initial provisioning}

Combining the characterization of $\bar{S}$, derived in Section 4, and the recursively derived closed-form formulas for the optimal order-up-to times, we characterize the optimal policy after initial provisioning in Theorem 5.1.

Theorem 5.1. (a) If $\mathrm{e}^{\lambda L} p /(p+h) \leqslant 1$, then $\bar{S}=0$ and the optimal order-up-to policy is $\pi\left(S_{0}^{*}, L_{0}^{*}\right)$, where $L_{0}^{*}=L+(c / p)$.

(b) If $1<\mathrm{e}^{\lambda L} p /(p+h) \leqslant 1+\lambda L$, then $\bar{S}=1$ and the optimal order-up-to policy is $\pi\left(S_{0}^{*}, L_{0}^{*}, L_{1}^{*}\right)$, where $L_{0}^{*}=L+(c / p)$, and

$$
\lambda L_{1}^{*}=\ln \left(1+\frac{\lambda d \mathrm{e}^{-\lambda L_{0}^{*}}+(h+p) \mathrm{e}^{-\lambda L}\left(1-\mathrm{e}^{-\lambda\left(L_{0}^{*}-L\right)}\right)}{p-(h+p) \mathrm{e}^{-\lambda L}}\right) .
$$

(c) If

$$
\sum_{k=0}^{m-1} \frac{(\lambda L)^{k}}{k !}<\mathrm{e}^{\lambda L} \frac{p}{p+h} \leqslant \sum_{k=0}^{m} \frac{(\lambda L)^{k}}{k !}, \quad m=(0,1,) 2, \ldots,
$$

then $\bar{S}=m$ and the optimal order-up-to policy is $\pi\left(S_{0}^{*}, L_{0}^{*}, L_{1}^{*}, \ldots, L_{m}^{*}\right)$, where $L_{0}^{*}=L+(c / p)$, $L_{1}^{*}$ is as given in part (b) of this theorem, and the optimal values for $L_{i}, i=2,3, \ldots, m$, can be determined recursively, using 


$$
\lambda L_{i}^{*}=\ln \left(1+\frac{\lambda c B_{i}+\lambda d C_{i}+(h+p)\left(D_{i}+E_{i}\right)-p A_{i}}{p-(h+p) D_{i}}\right) .
$$

Definitions of $A_{i}, B_{i}, C_{i}, D_{i}$, and $E_{i}$ are given in the previous subsection.

The only parameter of the optimal policy that remains to be determined is the initial order-up-to level $S_{0}^{*}$. This is done in Section 7. Next, in Section 6, a sensitivity analysis on the optimal order-up-to times is performed.

\section{Sensitivity analysis}

Since we have closed-form formulas for $L_{1}^{*}, L_{2}^{*}, \ldots, L_{\bar{S}}$, we are able to calculate them for any given values of the model parameters. Moreover, the calculations are not time consuming. Indeed, they are very fast. Consider, for instance, the following case:

- $c=2$. The price of a part doubles after the beginning of the final phase,

- $h=0.20$. The yearly holding cost rate is 0.20 ,

- $p=20$. The yearly backorder cost rate is 20 ,

- $d=0$. There is no removing cost or recycling revenue,

- $L=0.25$. The length of the replenishment lead time is three months,

- $\lambda=4$. Demand is Poisson distributed with a rate of 4 demands per year,

which we refer to as the basic case. The maximum order-up-to level is $\bar{S}=4$ in this case. The formulas for $L_{i}^{*}, i=0,1,2,3,4$, of Theorem 5.1 were programmed in Matlab for Windows, version 4.2c.1. We remark that the programming is straightforward and could be done for $i=5,6, \ldots$ also. It does get more tedious as $i$ increases, since the formula for $L_{i}^{*}$ becomes more lengthy. The calculations are very fast. On a PC with a Pentium 90 processor, we are able to calculate $L_{0}^{*}, \ldots, L_{4}^{*}$ in about three seconds. For the basic case this gives $L_{0}^{*}=0.35, L_{1}^{*}=0.04, L_{2}^{*}=0.13, L_{3}^{*}=0.33, L_{4}^{*}=0.66$.

In the remainder of this section, we report the results of a numerical sensitivity analysis. This analysis was performed by taking the basic case, and then separately varying each of the model parameters. This provides insight into the influence of the model parameters on the optimal order-up-to times, which the complex closed-form formulas themselves do not.

We observed many properties that are expected. If the replenishment cost $c$ (after time 0 ) increases, if the holding cost rate $h$ increases, if the disposal cost $d$ increases, or if the backorder cost rate $p$ decreases, then the optimal order-up-to times increase and hence the optimal order-up-to levels decrease. If the lead time $L$ increases or if the demand rate $\lambda$ increases, then the optimal order-up-to times decrease and hence the optimal order-up-to levels increase.

We also observed that the value of $h$ only has a minor influence on the optimal order-up-to times. The order-up-to times are mainly based on evaluating backorder cost against replenishment and disposal cost.

The optimal order-up-to times are very small if the disposal revenue is close to the ordering cost, i.e., if $d$ is close to $-c=-2$. This is expected since there is hardly any (cost-)risk of obsolescence in these cases. Due to the positive holding cost rate, the order-up-to times do remain positive.

\section{Optimal initial order-up-to level}

After having found the optimal order-up-to times, we next consider the determination of the optimal value $S_{0}^{*}$ for the initial order-up-to level $S_{0}$ at time 0 . This can be done similarly to the determination of the optimal base stock times in Section 5, which leads to Theorem 7.1. 
Theorem 7.1. (a) If $P \leqslant L+(1 / p)$, then $S_{0}^{*}=-\infty$.

(b) If $L+(1 / p)<P \leqslant L_{0}^{*}=L+(c / p)$, then $S_{0}^{*}$ is the largest positive integer $S_{0}$ for which

$$
1+d Q\left(S_{0}-1, \lambda P\right)+(h+p) E\left[\mathscr{T}_{1}\right]-p(P-L)<0,
$$

where

$$
E\left[\mathscr{T}_{1}\right]=S_{0} Q\left(S_{0}, \lambda L\right)-\lambda L Q\left(S_{0}-1, \lambda L\right)-Q\left(S_{0}, \lambda P\right)+\lambda P Q\left(S_{0}-1, \lambda P\right) .
$$

If (7) does not hold for any positive integer value of $S_{0}$, not even for $S_{0}=1$, then $S_{0}^{*}=0$.

(c) If $\sum_{k=0}^{i-1} L_{k}^{*}<P \leqslant \sum_{k=0}^{i} L_{k}^{*}$ for some $i=1,2, \ldots, \bar{S}$, or if $\sum_{k=0}^{i-1} L_{k}^{*}<P$ for $i=\bar{S}+1$, then $S_{0}^{*}$ is the largest integer $S_{0} \in\{i, i+1, \ldots\}$ for which

$$
\operatorname{Pr}\left(\mathscr{E}_{1}\right)+d \operatorname{Pr}\left(\mathscr{E}_{2}\right)+(h+p) E\left[\mathscr{T}_{1}\right]-p E\left[\mathscr{T}_{1}+\mathscr{T}_{2}\right]<0,
$$

where

$$
\begin{aligned}
\operatorname{Pr}\left(\mathscr{E}_{1}\right)= & \sum_{n_{1, i} \in V_{i+1}\left[S_{0}-i\right]}\left[\prod_{j=1}^{i} q\left(n_{j}, \lambda t_{j}\right)\right], \\
\operatorname{Pr}\left(\mathscr{E}_{2}\right)= & \sum_{n_{1, i} \in V_{i+1}\left[S_{0}-i\right]}\left[\left(\prod_{j=1}^{i} q\left(n_{j}, \lambda t_{j}\right)\right) Q\left(S_{0}-1-\sum_{j=1}^{i} n_{j}, \lambda t_{i+1}\right)\right], \\
\lambda E\left[\mathscr{T}_{1}\right]= & S_{0} Q\left(S_{0}, \lambda L\right)-\lambda L Q\left(S_{0}-1, \lambda L\right) \\
& -\sum_{k=1}^{i-1} \sum_{n_{1, k} \in V_{k}\left[S_{0}-i\right]}\left(\prod_{j=1}^{k} q\left(n_{j}, \lambda t_{j}\right)\right)\left(\begin{array}{c}
(i-k) Q(i-k, \lambda L) \\
-\lambda L Q(i-k-1, \lambda L)
\end{array}\right) \\
& -\sum_{n_{1, i} \in V_{i+1}\left[S_{0}-i\right]}\left(\prod_{j=1}^{i} q\left(n_{j}, \lambda t_{j}\right)\right)\left(\begin{array}{c}
\left(S_{0}-\sum_{j=1}^{i} n_{j}\right) Q\left(S_{0}-\sum_{j=1}^{i} n_{j}, \lambda t_{i+1}\right) \\
-\lambda t_{i+1} Q\left(S_{0}-1-\sum_{j=1}^{i} n_{j}, \lambda t_{i+1}\right)
\end{array}\right), \\
\lambda E\left[\mathscr{T}_{2}\right]= & \sum_{k=1}^{i} \sum_{n_{1, k} \in V_{k}\left[S_{0}-i\right]}\left(\prod_{j=1}^{k} q\left(n_{j}, \lambda t_{j}\right)\right)\left(k+S_{0}-i\right) \\
& +\sum_{n_{1, i} \in V_{i+1}\left[S_{0}-i\right]}\left(\prod_{j=1}^{i} q\left(n_{j}, \lambda t_{j}\right)\right)\left(\lambda\left(t_{i+1}-L\right)+\sum_{j=1}^{i} n_{j}\right), \\
t_{1}= & P-\sum_{k=0}^{i-1} L_{k}^{*}, \quad L_{i+1-j}^{*}, \quad j=2,3, \ldots, i+1 .
\end{aligned}
$$

See also Section 5.2 for notations. If $(8)$ does not hold for any integer value of $S_{0} \geqslant i$, not even for $S_{0}=i$, then $S_{0}^{*}=i-1$.

Proof 7.1. (a) Similarly to the derivation of $L_{0}^{*}$ in Section 5. (b) Since $P \leqslant L_{0}^{*}$, Theorem 5.1 gives that no parts will be ordered after time 0 . We compare the policies $\pi\left(S_{0}, L_{0}^{*}\right)$ and $\pi\left(S_{0}-1, L_{0}^{*}\right)$, which we denote for short by $\pi\left(S_{0}\right)$ and $\pi\left(S_{0}-1\right)$. It is assumed that the stock just before time 0 is at most $S_{0}-1$, so that policy $\pi\left(S_{0}\right)$ orders one more part at that time.

The optimal provisioning $S_{0}^{*}$ is expected to be the largest value of $S_{0} \geqslant 1$ for which policy $\pi\left(S_{0}\right)$ gives lower expected cost than $\pi\left(S_{0}-1\right)$; it is 0 if policy $\pi(1)$ gives higher expected cost than $\pi(0)$. 
The difference in expected cost between policies $\pi\left(S_{0}\right)$ and $\pi\left(S_{0}-1\right)$ is

$$
\operatorname{Pr}\left(\mathscr{E}_{1}\right)+d \operatorname{Pr}\left(\mathscr{E}_{2}\right)+(h+p) E\left[\mathscr{T}_{1}\right]-p E\left[\mathscr{T}_{1}+\mathscr{T}_{2}\right],
$$

where the events $\mathscr{E}_{1}, \mathscr{E}_{2}$ and the random variables $\mathscr{T}_{1}, \mathscr{T}_{2}$ are defined as before (see Section 5.2.2).

Clearly, $\operatorname{Pr}\left(\mathscr{E}_{1}\right)=1$, and $\operatorname{Pr}\left(\mathscr{E}_{2}\right)=\operatorname{Pr}\left(M_{S_{0}}>P\right)$, where $M_{S_{0}}$ denotes the moment at which the $S_{0}$ th arrival after time 0 occurs. Since demand follows a Poisson process with rate $\lambda$, this gives $\operatorname{Pr}\left(\mathscr{E}_{2}\right)=Q\left(S_{0}-1, \lambda P\right)$. It remains to be shown that the expressions for $E\left[\mathscr{T}_{1}\right]$ and $E\left[\mathscr{T}_{1}+\mathscr{T}_{2}\right]$ are correct. It is easy to see that

$$
E\left[\mathscr{T}_{1}\right]=E\left[M_{S_{0}}-L\right]^{+}-E\left[M_{S_{0}}-P\right]^{+},
$$

which can be rewritten (see [18, Appendix B]) as the expression given in the theorem. Finally, the expression for $E\left[\mathscr{T}_{1}+\mathscr{T}_{2}\right]$ is correct, since the difference in stock on hand minus the number of backorders between policies $\pi\left(S_{0}\right)$ and $\pi\left(S_{0}-1\right)$ is one part in period $[L, P]$.

(c) The proof is similar to that of part (b) of this theorem. We refer the interested reader to [18].

\section{Limitations of the proposed method and directions for future extensions}

There are several interesting extensions of the model that we presented: (a) non-stationary demand, (b) a positive setup cost for ordering, (c) a stochastic lead time, (d) present value considerations, and (e) single depot versus central depot and repair kits. Below, we will shortly discuss these extensions, and indicate whether the method that we presented could be modified to analyze them.

(a) The demand rate for service parts can be increasing with time if older appliances have higher failure rates, and decreasing with time if the population of appliances in use decreases. Only in the latter case, the ordering policy that we proposed with a decreasing order-up-to level still seems appropriate. But our method of optimization cannot (easily) be modified for a non-stationary Poisson demand process.

(b) The ordering policy that we proposed can be modified for situations with a positive setup cost for ordering by including time-dependent order levels. We expect that a method similar to the one that we presented can again be used to calculate the optimal policy, though the analysis will surely be a lot more complicated.

(c) and (d) The policy that we proposed still seems appropriate. However, our method of optimization cannot (easily) be modified for these extensions of our model.

(e) Demands for service parts often occur at many different customer sites that are visited by repairmen. These repairmen carry a repair kit, containing a selection of service parts, with them. So, service parts are stocked in a central depot and in repair kits. Our method for finding the optimal order-up-to policy can be modified (under certain assumptions) for these situations. We will present the results in the near future.

\section{Conclusion}

We considered an appliance manufacturer's problem of controlling the inventory of a service part in its final phase. The planning horizon was deterministic and known, and the objective was to minimize the total expected undiscounted costs of replenishment (no setup cost), inventory holding, backorder, and disposal (of unused parts at the end of the planning horizon).

We proposed an ordering policy consisting of an initial order-up-to level at time 0 , and a subsequent series of decreasing order-up-to levels for various intervals of the planning horizon. See Fig. 2. We presented a method of calculating the optimal policy. Indeed, we were able to describe the optimal policy using a set of closed-form formulas. Though these formulas are complicated, programming them in a software 
package (we used Matlab) is straightforward. Once that has been done, the optimal policy can be calculated very fast. As a result, it is also easy to perform sensitivity analysis.

In the future we will extend the method to situations with a central depot and repair kits rather than a single depot. Moreover, we will try to extend the policy and the method to situations with a positive setup cost for ordering.

\section{References}

[1] L.C. Barbosa, M. Friedman, Inventory lot size models with vanishing market, Journal of the Operational Research Society 30 (12) (1979) 1129-1132.

[2] E.V. Denardo, Dynamic Programming: Models and Applications, Prentice-Hall, Englewood Cliffs, NJ, 1982.

[3] L. Fortuin, The all-time requirement of spare parts for service after sales - theoretical analysis and practical results, International Journal of Operations and Production Management 1 (1) (1980) 59-69.

[4] L. Fortuin, Reduction of the all-time requirement of spare parts, International Journal of Operations and Production Management 2 (1) (1981) 29-37.

[5] J.H.J. Geurts, J.M.C. Moonen, On the robustness of 'insurance type' spares provisioning strategies, Journal of the Operational Research Society 43 (1992) 43-51.

[6] D. Iglehart, Dynamic programming and stationary analysis of inventory problems, in: H.E. Scarf, D.M. GilfordM.W. Shelly (Eds.), Multistage Inventory Models and Techniques, Stanford University Press, Stanford, CA, 1963 (Chapter 1).

[7] D. Iglehart, Optimality of $(s, S)$ policies in the infinite horizon dynamic inventory problem, Management Science 9 (1963) 259267.

[8] W.K. Klein Haneveld, R.H. Teunter, Optimal provisioning strategies for slow moving spare parts with small lead times, Journal of the Operational Research Society 48 (2) (1997) 184-194.

[9] S. Nahmias, Perishable inventory theory: A review, Operations Research 30 (4) (1982) 680-708.

[10] E.L. Porteus, On the optimality of generalized $(s, S)$ policies, Management Science 17 (1971) 411-426.

[11] F. Raafat, Survey of literature on continuous deteriorating inventory models, Journal of the Operational Research Society 42 (1991) 27-37.

[12] H. Scarf, The optimality of $(S, s)$ policies in the dynamic inventory problem, in: K. Arrow, S. Karlin, P. Suppes (Eds.), Mathematical Models in the Social Sciences, Stanford University Press, Stanford, CA, 1960.

[13] E.A. Silver, R. Peterson, Decision Systems for Inventory Management and Production Planning, Wiley, New York, 1985.

[14] R.H. Teunter, Inventory control of service parts in the final phase, Ph.D. Thesis, University of Groningen (RUG), The Netherlands, 1998.

[15] R.H. Teunter, L. Fortuin, End-of-life service: A case study, European Journal of Operational Research 107 (1) (1998) 19-34.

[16] R.H. Teunter, L. Fortuin, End-of-life service, International Journal of Production Economics 59 (1999) $487-497$.

[17] R.H. Teunter, W.K. Klein Haneveld, The 'final order' problem, European Journal of Operational Research 107 (1) (1998) 35-44.

[18] R.H. Teunter, W.K. Klein Haneveld, Inventory control of service parts in the final phase, Extended version including all proofs, 2000.

[19] H.C. Tijms, Analysis of $(s, S)$ inventory models, Mathematical Centre Tracts 95 (1972).

[20] A.F. Veinnot Jr., On the optimality of $(s, S)$ inventory policies: New conditions and a new proof, SIAM Journal on Applied Mathematics 14 (1966) 1067-1083. 